\title{
Sustainability in static-priority restricted-migration scheduling
}

\author{
Frédéric Fauberteau \\ CEA, List, \\ Embedded Real-Time Systems Laboratory \\ Point Courrier 94 \\ Gif-sur-Yvette, F-91191 France \\ frederic.fauberteau@cea.fr
}

\author{
Serge Midonnet \\ Université Paris-Est \\ LIGM, UMR CNRS 8049 \\ 5, bd Descartes - Champs-sur-Marne \\ 77454 Marne-la-Vallée CEDEX 2, France \\ serge.midonnet@univ-paris-est.fr
}

\begin{abstract}
In this paper, we focus on the static-priority scheduling of periodic hard real-time tasks upon identical multiprocessor platforms. In order to bound the inter-processor migrations, we consider the restricted-migration scheduling policy for which a task is allowed to migrate only at job boundaries. Several jobs of the same task can then be assigned on different processors but a given job can not migrate. It has been shown that this scheduling policy can suffer from scheduling anomalies. These anomalies occur when a decrease in execution requirement of a job causes a deadline miss. We present a static-priority restricted-migration scheduling algorithm and we prove it does not suffer from these anomalies. We also review the scheduling anomalies according to the scheduling tests for this algorithm.
\end{abstract}

\section{Categories and Subject Descriptors}

C.3 [Special-purpose and application-based systems]: Real-time and embedded systems; J.7 [Computers in other systems]: Real time

\section{General Terms}

Algorithms

\section{Keywords}

Real-Time, Scheduling, Multiprocessor, Sustainability

\section{INTRODUCTION}

The recent improvements in the embedded systems induce more powerful and complex systems on which we can reasonably run a modern operating system. In the case of the realtime embedded applications, a Real-Time Operating System (RTOS) can be a good solution to provide security and reliability. A RTOS also enables to implement an advanced scheduler without suffering from intolerable overrides due

Permission to make digital or hard copies of all or part of this work for personal or classroom use is granted without fee provided that copies are not made or distributed for profit or commercial advantage and that copies bear this notice and the full citation on the first page. To copy otherwise, to republish, to post on servers or to redistribute to lists, requires prior specific permission and/or a fee.

RACS'12 October 23-26, 2012, San Antonio, TX, USA.

Copyright 2012 ACM 978-1-4503-1492-3/12/10 ...\$10.00. to costly computations of scheduling decisions. In the case of the multiprocessor embedded systems, the choice of the scheduler is very important in order to reduce the number of context switches and migrations.

The literature about real-time multiprocessor scheduling often states two main approaches (a third one is presented bellow) to schedule sets of tasks. These two multiprocessor scheduling approaches are referenced as global and partitioned scheduling. The first one allows inter-processor migrations while the second one prevents them. Although the superiority of partitioned scheduling over global scheduling has been highlighted according to the best known schedulability tests [2], these two approaches are incomparable. Some set of tasks can be scheduled with partitioned algorithm and not by a global one and vice versa. It remains difficult to choose among one of these two approaches. As presented in [7], the intermediate restricted-migration approach has to be considered. This approach allows task-level migrations but no job-level migrations. A non-hybrid ${ }^{1}$ multiprocessor scheduling algorithm can be classified among one of the following classes:

- partitioned: Each task is assigned on a processor. For a given task, each job is released on the processor on which the task has been assigned. No migration is allowed (e.g. [19], EDF-FF [17]).

- restricted-migration: At each time a job is released, it can be assigned on a different processor. The tasklevel migrations are allowed but a job can not migrate during its execution (e.g. $r-E D F[5], r-S P_{-} w l[8]$ ).

- global: The job-level migrations are allowed. A job can start on a first processor and finishes on a second one (e.g. RM-US[m/(3m-2)] [1]), PriD [12]).

This classification reminds the one made for the three main classes of uniprocessor scheduling algorithm. A uniprocessor scheduling algorithm can be classified among one of the following classes:

- fixed-task-priority or static-priority: A priority is attributed to each task composing the system. For a given task, each job inherits the priority of the task which generates it. The priority of a job is constant during its execution. (e.g. Rate-Monotonic [16], Deadline-Monotonic [15], ... ).

\footnotetext{
${ }^{1}$ An hybrid algorithm exploits behavior from two different classes for two different subsets of tasks (See [11] for more details)
} 
- fixed-job-priority: At each time a job is released, its priority is computed and it is constant during the execution of the job (e.g. Earliest-Deadline-First [16]).

- dynamic-priority: The priority of any job can change during its execution (e.g. Least-Laxity-First [18])

We refer to a fixed-priority scheduling algorithm in the case where the scheduling algorithm is either fixed-taskpriority or fixed-job-priority and only jobs are considered.

In multiprocessor context, a non-hybrid scheduling algorithm can reasonably be referred according to both how the prioritization is done (fixed-task / fixed-job / dynamic) and how the migrations are allowed (no / task-level / job-level).

Although the restricted-migration scheduling has been less studied than the two main multiprocessor scheduling approaches, we can exhibit some interesting works. Ha and Liu have studied the fixed-priority restricted-migration scheduling of a set of jobs and have shown this kind of scheduling is prone to a problem of predictability (scheduling anomaly which occurs when the execution requirement of jobs is decreased). Baruah and Carpenter have proposed $r-E D F$, an EDF-based algorithm for periodic tasks on identical processors [5]. In the same paper, they also proposed an extension of PriD [12] to $r$-PriD (which exploits restricted-migration). Funk has studied in her thesis [10] the restricted-migration scheduling approach in the case of an uniform multiprocessor. Fisher has studied in his thesis [9] the restrictedmigration scheduling approach in the case of scheduling of a set of jobs. Fauberteau et al. have proposed $r-S P_{-} w l$, a static-priority algorithm for periodic tasks on identical processors [8]. The contribution of this paper is to propose a proof of sustainability for $r-S P \_w l$ algorithm in the case of the constrained deadline period sets of tasks.

The remainder of this paper is organized as follows. In Section 2, we remind the model that we consider and we introduce the notations that we use. In Section 3, we define the concept of sustainability and we give an example illustrating a scheduling anomaly which can occur with a non-predictable algorithm. In Section 4, we present our static-priority restricted-migration scheduling algorithm and we prove that it is sustainable according to our considered model. We conclude in Section 5 by giving some future work perspectives.

\section{SYSTEM MODEL}

We consider a set $\tau$ of $n$ constrained deadline periodic tasks indexed from 1 to $n$. (i.e. $\left.\tau=\left\{\tau_{1}, \ldots, \tau_{n}\right\}\right)$. The index of the task is in inverse proportion to its priority. The less is the task index the higher its priority is.

A task $\tau_{i}$ is characterized by:

- its Worst Case Execution Time (WCET) denoted $C_{i}$,

- its relative deadline denoted $D_{i}$,

- its period denoted $T_{i}$.

A task $\tau_{i}\left(C_{i}, D_{i}, T_{i}\right)$ consists of an infinite recurrence of jobs where each job $J_{i, k}$ is characterized by:

- its release time denoted $r_{i, k}$,

- its execution requirement denoted $e_{i, k}$ such that $0 \leq$ $e_{i, k} \leq C_{i}$
- its absolute deadline denoted $d_{i, k}$ such that $d_{i, k}=$ $r_{i, k}+D_{i}$.

For readability, we can consider a job independently of the task which generates it and we denote it $J_{k}\left(r_{k}, e_{k}, d_{k}\right)$.

By definition, the utilization of a periodic task is given by $u_{i}=\frac{C_{i}}{T_{i}}$. By extension, we define the utilization of a set of tasks as the sum of the utilization of the tasks which compose it and its value is given by $U(\tau)=\sum_{i}^{n} u_{i}$. We also denote $U_{\max }(\tau)$ the maximum utilization of $\tau$ and we define it as: $U_{\max }(\tau)=\max _{i}^{n} u_{i}$.

We also consider a set $\Pi$ of $m$ processors $\pi_{j}$ indexed from 1 to $m$ (i.e. $\left.\Pi=\left\{\pi_{1}, \ldots, \pi_{m}\right\}\right)$. We consider the processors as identical (homogeneous case). In other terms, each job is executed as the same rate on any processor.

\section{SUSTAINABILITY}

In order to analyze a given hard real-time system, a technique consists in highlighting the worst case scenario in which the worst response time could be met. Intuitively, this scenario has to occur when the utilization of the set of tasks is maximum. In other words, it has to occur when the execution requirement of each job is maximum and when the inter-arrival time of each task is minimum.

But it has been shown that some classes of algorithms are prone to scheduling anomalies $[14,13]$. In particular, it has been shown that a fixed-priority restricted-migration job assignment is not predictable. Let $\mathcal{J}_{k}$ denote a job generated by a task $\tau_{i}$. Let $\mathcal{S}_{k}$ denote the start time of $\mathcal{J}_{k}$ and $\mathcal{S}_{k}^{-}$its minimum start time. In the same manner, let $\mathcal{F}_{k}$ denote the finish time of $\mathcal{J}_{k}$ and $\mathcal{F}_{k}^{+}$its maximum finish time. The non-predictability of a scheduling algorithm is a scheduling anomaly which occurs when the execution time $e^{k}$ of $\mathcal{J}_{k}$ is such that $e_{k}^{-} \leq e_{k} \leq e_{k}^{+}$and (i) either $\mathcal{S}_{k}<\mathcal{S}_{k}^{-}$or (ii) $\mathcal{F}_{k}>\mathcal{F}_{k}^{+}$.

In Figure 1, we show an example from [14] illustrating this kind of scheduling anomaly. The Table 1(a) represents the arrival time $r_{k}$, the absolute deadline $d_{k}$ and the execution requirement $e_{k}$ of the job $J_{k}$ for $1 \leq k \leq 6$. The priority of the jobs are given in decreasing order of their index $\left(J_{1}\right.$ is the higher priority and $J_{6}$ is the lower priority). In this example, the execution requirement of $J_{2}$ can vary from 2 to 6 . In Figure 1(b), respectively $1(\mathrm{c}), J_{2}$ runs for its maximum, respectively its minimum, execution requirement. We notice that all deadlines are met. Intuitively, we could conclude that no deadlines can be missed in all cases. But in Figure $1(\mathrm{~d}), J_{2}$ runs for 3 time units and $J_{4}$ misses its deadline. Finally, in Figure 1(e), when $J_{2}$ runs for 5 time units, the finish time $\mathcal{F}_{4}$ of $J_{4}$ is minimum.

The predictability is a notion linked to the execution requirement of the jobs. It consists in guaranteeing that a scheduling algorithm can successfully schedule a set of jobs when the execution requirement of one or many jobs is reduced. The concept of sustainability comes from the notion of predictability but it is extended to the relaxation of other parameters as relative deadlines or arrival times.

Definition 1 (Sustainable Algorithm). Let $\mathcal{A} d e-$ note a scheduling algorithm. Let $\tau$ denote any set of tasks that is $\mathcal{A}$-schedulable. Let $\mathcal{J}$ denote a collection of jobs generated by $\tau$. Scheduling algorithm $\mathcal{A}$ is said to be sustainable if and only if $\mathcal{A}$ meets all deadlines when scheduling any collection of jobs obtained from $\mathcal{J}$ by changing the parameters 


\begin{tabular}{|c||c|c|c||c||c|c|c|}
\hline job & $r_{k}$ & $d_{k}$ & {$\left[e_{k}^{-}, e_{k}^{+}\right]$} & job & $r_{k}$ & $d_{k}$ & {$\left[e_{k}^{-}, e_{k}^{+}\right]$} \\
\hline \hline$J_{1}$ & 0 & 10 & {$[5,5]$} & $J_{4}$ & 0 & 20 & {$[10,10]$} \\
$J_{2}$ & 0 & 10 & {$[2,6]$} & $J_{5}$ & 5 & 200 & {$[100,100]$} \\
$J_{3}$ & 4 & 15 & {$[8,8]$} & $J_{6}$ & 7 & 25 & {$[2,2]$} \\
\hline
\end{tabular}

(a) Job parameters

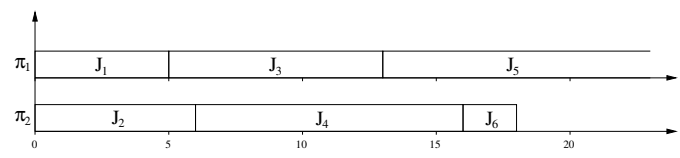

(b) $e_{2}=6$

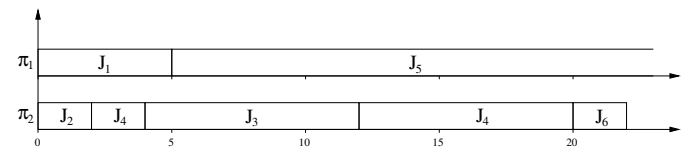

(c) $e_{2}=2$

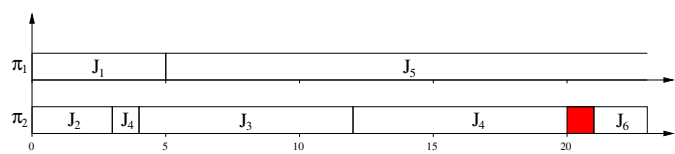

(d) $e_{2}=3$

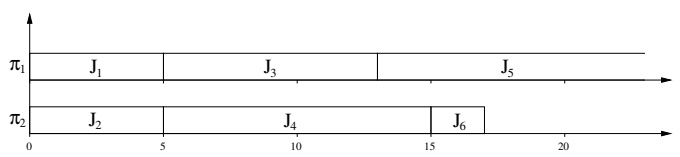

(e) $e_{2}=5$

Figure 1: A example illustrating scheduling anomalies from [14].

of one or more individual jobs in any, some, or all the following ways: (i) decreased execution requirements; (ii) larger relative deadlines and (iii) latter arrival times.

In addition to the scheduling algorithms, the concept of sustainability can be used in the case of schedulability tests. In the same manner a scheduling policy can suffer from scheduling anomalies when job or task parameters are relaxed, a schedulability test can argue that a set of tasks is schedulable and invalidate this response if one or many of the scheduling parameters is relaxed.

Definition 2 (Sustainable test). Let $\mathcal{A}$ denote $a$ scheduling algorithm and $\mathcal{F}$ a schedulability test for $\mathcal{A}$. Let $\tau$ denote any set of tasks that is $\mathcal{A}$-schedulable. Let $\mathcal{J}$ denote a collection of jobs generated by $\tau$. Scheduling test $\mathcal{F}$ is said to be sustainable if and only if $\mathcal{A}$ meets all deadlines when scheduling any collection of jobs obtained from $\mathcal{J}$ by changing the parameters of one or more individual jobs in any, some, or all the following ways: (i) decreased execution requirements; (ii) larger relative deadlines and (iii) latter arrival times.

In the case of uniprocessor scheduling, the schedulability tests have been studied in order to highlight which of them are sustainable $[4,6]$. This work has been extended in the case of the multiprocessor scheduling [3].

\section{SCHEDULING ALGORITHM}

In Section 4.1 below, we describe the behavior of the fixed-priority restricted-migration scheduling algorithm as presented in [14]. In Section 4.2, we define $r-S P_{-} w l$, a staticpriority restricted-migration multiprocessor scheduling algorithm for scheduling periodic sets of tasks upon identical multiprocessor platforms. In Section 4.3, we prove that $r$ $S P \_w l$ is sustainable according to decreased execution time, later arrival times and larger relative deadlines. In Section 4.4 , we review the sustainability of the schedulability tests of $r$-SP_wl.

\subsection{Non-predictable algorithm}

We have reminded in Section 3 that a fixed-priority restricted-migration scheduling algorithm is not predictable. By extension, a static-priority restricted-migration suffers from the same scheduling anomaly. In order to propose a predictable scheduling algorithm of this class, it is necessary to analyze the properties of the scheduling algorithm given in [14] to discover which property is responsible of the nonpredictability. This scheduling algorithm is described by the Algorithm 1.

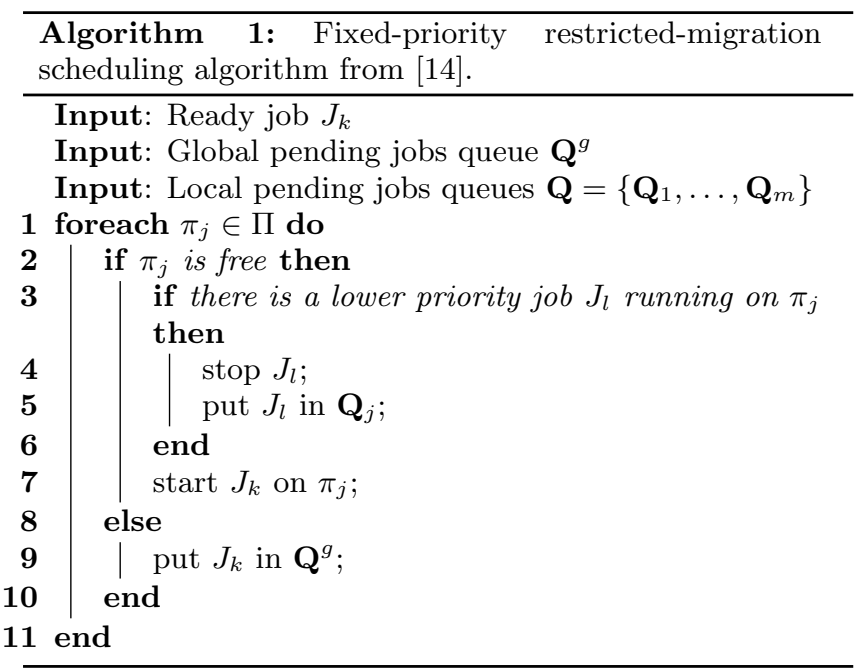

This algorithm is designed to assign jobs on the multiprocessor platform when they are released only if a processor is available. In this last case, the assignment is postponed to a time at which a processor becomes available (the finish time of a higher priority job). The ready job $J_{k}$ is tested to be assigned on one of the $m$ available processors (Lines 1-11). If one of these processors is free - in the sense of no higher priority job is running on this processor - then $J_{k}$ starts on $\pi_{j}$ (Lines 2-7). If a lower priority job is already running, it is preempted and put in the $\pi_{j}$ local queue (Lines 3-6). Otherwise, if no processor is free for $J_{k}$ at its release time, its start is postponed and it is put in the global queue $\mathbf{Q}^{g}$ (Lines 8-9). For readability, we voluntarily omit the algorithm part corresponding to the finish time of a job. But when a job finishes its execution, the scheduler is called to check the pending local and global job queues in order to start the higher priority waiting job.

DEFINITION 3 (LOWER-PRIORITY-AGNOSTIC TEST). A schedulability test is lower-priority-agnostic if for a given job $J_{k}$, no lower priority than $J_{k}$ job has to be considered to decide the schedulability of $J_{k}$.

As shown in Figure 1(d), the fixed-priority restrictedmigration scheduling algorithm from [14] is lower-priority- 
agnostic since $J_{3}$ is assigned on processor $\pi_{2}$ without consideration in $J 4$ which will miss deadline because of lake of processor execution time.

Definition 4 (Processor-AVAilable Assignment). An assignment is processor-available if the assignment of a job $J_{k}$ is made when a processor becomes available.

As shown in Figures 1(b) and 1(c), the algorithm from [14] performs a processor-available assignment. The job $J_{3}$ released at time 4 is either assigned on processor $\pi_{1}$ at time 5 because no processor is available before or on processor $\pi_{2}$ at time 4 because of lower priority job runs and can be preempted.

Definition 5 (Work-CONSERVING ALGORITHM). A multiprocessor scheduling algorithm is work-conserving if no processor is idled while there are active jobs.

As explained above, when a job finishes its execution, the scheduler is executed in order to wake up a potential pending job from both the processor local queues and the global system queue. This behavior leads to produce a workconserving scheduler.

We have shown that the fixed-priority restricted-migration scheduling algorithm from [14] is a work-conserving algorithm and that its job assignment is processor-available using a schedulability test which is lower-priority-agnostic.

\subsection{Algorithm $r-S P_{-} w l$}

According to the previous cited properties, we now examine how to design a predictable static-priority restrictedmigration scheduling algorithm. In a more general purpose, we extend this property of predictability to a sustainability one.

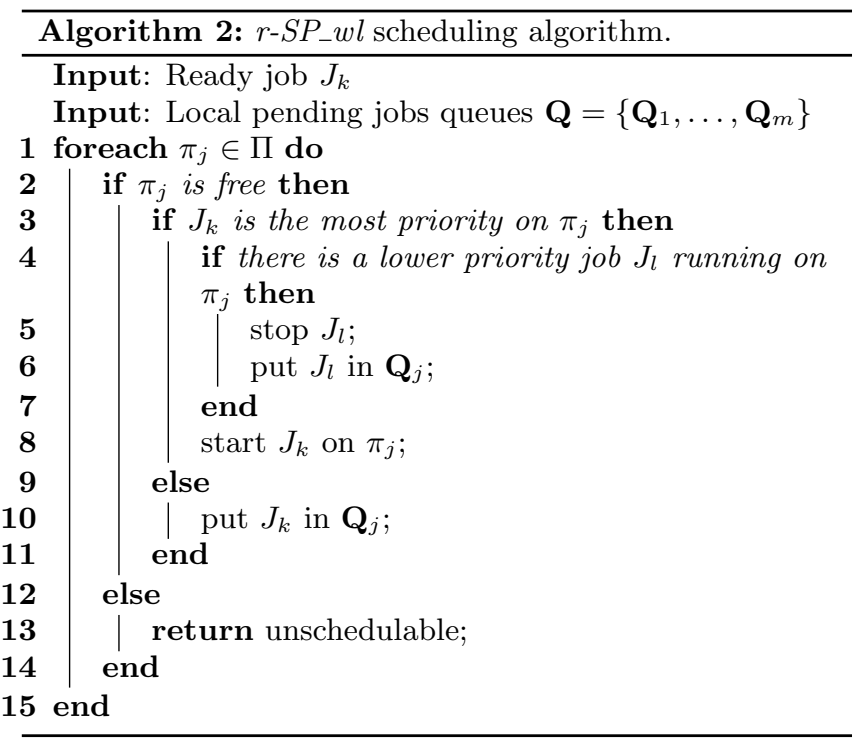

This algorithm is designed to assign jobs on the multiprocessor platform at their release time even if no processor is available. In this last case, jobs are put in the local queue of the chosen processor. The ready job $J_{k}$ is tested to be assigned on one of the $m$ available processor (Lines 1-15). If one of these processors is free then $J_{k}$ starts on $\pi_{j}$ (Lines 211). With $r$-SP_wl, a processor $\pi_{j}$ is considered free for the job $J_{k}$ if all jobs $J_{j} \in J\left(\pi_{j}\right)^{\prime}$ (with $J\left(\pi_{j}\right)^{\prime}=J\left(\pi_{j}\right) \cup J_{k}$ ) have enough laxity $L_{j}\left(\pi_{j}, r_{k}\right)$ on processor $\pi_{j}$ at their released time $r_{j}$ to be scheduled. The laxity values are computed and maintained by the scheduler. If a lower priority job is already running, it is preempted and put in the $\pi_{j}$ local queue (Lines 3-8). If no processor is free for job $J_{k}$ at its release time, it is considered unschedulable. Like the algorithm presented in [14], the scheduler has to be executed at a time when a job finishes its execution but it has only to examine the local queue of the processor on which this job finishes. The laxity has also to be decreased for a job $J_{k}$ at time $r_{k}+C_{k}$ in order to guarantee the predictability of the schedule.

DEFINITION 6 (LAXITY). The laxity $L_{k}\left(\pi_{j}, t\right)$ of a job $J_{k}$ on a processor $\pi_{j}$ at time $t$ is given by:

$$
L_{k}\left(\pi_{j}, t\right)=D_{k}-C_{k}-\sum_{J_{h} \in h p\left(\pi_{j}, J_{k}\right)} e_{h}^{*}(t)
$$

where $e_{h}^{*}(t)$ denotes the remaining execution requirement of the job $J_{h}$ at time $t$.

A static-priority schedulability test is in general lowerpriority-agnostic. In uniprocessor, this behavior is completely justified because a higher priority job has to be scheduled before other jobs even if these last ones do not meet their deadline. But in the multiprocessor case, this behavior can lead to the case that the job is assigned on a processor on which a lower priority task could miss its deadline or migrate while another processor could discard this eventuality. In the case of a global scheduler, a lower-priority-agnostic test can lead to a more important number of migrations without impacting the schedulability. But in the case of restricted-migration approach, it can cause deadline misses.

Definition 7 (LOWER-PRIORITY-AWARE TEST). $A$ schedulability test is lower-priority-aware if for a given job $J_{k}$, lower priority than $J_{k}$ jobs have to be considered to decide the schedulability of $J_{k}$.

In the case of fixed-priority restricted-migration, one of the main causes of the predictability problem is the processoravailable assignment. During the release time of a job and when a processor becomes free to admit it, many changes can occur in the scheduling scenario. These changes can lead to the job assigned on a completely different processor. At this point, it is impossible to predict the worst case behavior of this job since it is not allowed to migrate on another processor in order to continue its execution after a preemption by a higher priority job.

Definition 8 (RElEASE-Time ASSignment). An assignment is release-time if the assignment of a job $J_{k}$ is made when $J_{k}$ becomes active (at its release time) instead of the time at which a processor becomes available.

The algorithm $r$-SP_wl is driven by the laxity of the jobs at time $t$. We can notice that this value of laxity is computed using the WCET of the task which generates the job. If a job $J_{k}$ finishes its execution earlier than this WCET, it can result in an idle time on the processor on which $J_{k}$ was executed.

Definition 9 (IDle Algorithm). A multiprocessor scheduling algorithm is idle if at least one processor can be idled while there are active jobs. 


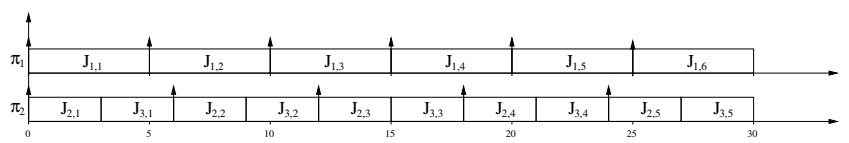

(a) Schedulable set of jobs.

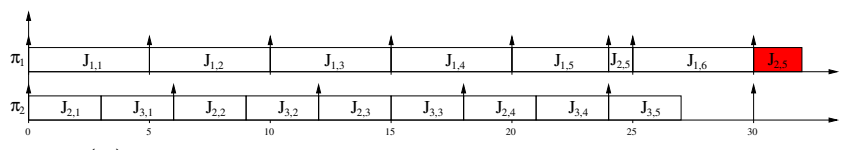

(b) Unschedulable set of jobs without idle times.

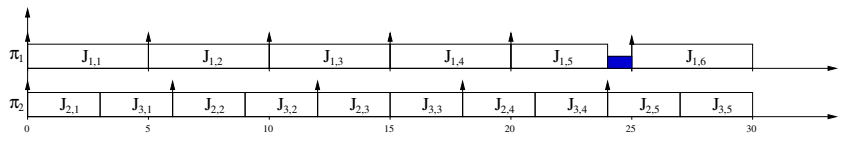

(c) Schedulable set of jobs with idle times.

Figure 2: Impact of idle times in restrictedmigration scheduling.

We illustrate in Figure 2 the problems which we can encounter with restricted-migration scheduling in the case of work-conserving algorithms and lower-priority-agnostic schedulability tests. The system depicted in this figure is generated by three tasks $\tau_{1}(5,5,5), \tau_{2}(3,6,6)$ and $\tau_{3}(3,6,6)$ and scheduled among two processors. In Figure 2(a), we represent a set of jobs schedulable with respect to the staticpriority restricted-migration scheduling. In Figure 2(b), we represent the same set of jobs but the job $J_{1,5}$ has an execution requirement of 4 instead of 5 . In this case, the processor $\pi_{1}$ becomes idle at time 24 . In this scenario, the job $J_{2,5}$ can be assigned to the processor $\pi_{1}$ and start its execution. But at time 25 , the sixth job of $\tau_{1}$ is released and preempts the job of $\tau_{2}$ since $\tau_{1}$ is higher priority. At this time, it is possible to know that $J_{2,5}$ will miss its deadline because it has not enough laxity to finish its execution. In Figure 2, the same set of jobs is depicted, but the lower-priority-aware test of $r-S P \_w l$ is used. Since the laxity of a job on a processor is updated at the time of its worst case finish time, the laxity of $J_{1,5}$ at time 24 is always 0 and therefore the laxity of $J_{2,5}$ is also 0 . The processor $\pi_{1}$ can not be considered as available for the assignment of this last job. This behavior leads to produce idle times in scheduling. It is also important to notice that the lower-priority-aware schedulability test of $r$-SP_wl does not allow $J_{1,6}$ to be assigned on $\pi_{1}$ because the laxity of lower priority job $J_{2,6}$ would not be enough to permit it to finish its execution before its deadline.

We shown that $r$-SP_wl differs from the fixed-priority restricted-migration from [14] in three points. We now prove that it is sustainable.

\subsection{Sustainability of $r-S P_{-} w l$}

In order to prove the sustainability of $r-S P_{-} w l$, we must verify that it can successfully schedule a set of tasks according to the three relaxations of parameters which are (i) execution requirement decreasing (ii) relative deadline increasing (iii) arrival time increasing. We verify these points by the three following lemmas.

LEMma 1. Let $\tau$ denote any set of tasks that is schedulable by r-SP_wl. Let $\mathcal{J}$ denote a collection of jobs generated by $\tau$ The algorithm $\mathrm{r}-\mathrm{SP}$-wl meets all deadlines when scheduling any collection of jobs obtained from $\mathcal{J}$ by changing the parameters of one or more jobs by decreasing execution requirements.

Proof. The proof comes from a mathematical induction over the priority of the jobs. Let $J$ be a set of $n$ jobs. We denote $J_{i}^{-}$the job for which the execution requirement is such that $e_{i}^{-}<e_{i}$. We also denote $L_{i}^{-}\left(\pi_{j}, t\right)=D_{i}-C_{i}-$ $\sum_{J_{h}^{-} \in h p\left(\pi_{j}, J_{i}^{-}\right)} e_{j}^{-*}(t)$ the laxity of $J_{i}^{-}$on the processor $\pi_{j}$ at time $t$. The induction hypothesis is $\forall 1 \leq i \leq n, L_{i}^{-}\left(\pi_{j}, t\right) \geq$ $L_{i}\left(\pi_{j}, t\right)$. The hypothesis is true at step one by definition of the laxity since $J_{i}$ is the most priority job: $L_{i}^{-}\left(\pi_{j}, t\right)=$ $L_{i}\left(\pi_{j}, t\right)$. We suppose the induction is true at step $i$. We verify that the relation $L_{i+1}^{-}\left(\pi_{j}, t\right) \geq L_{i+1}\left(\pi_{j}, t\right)$ is true for the job $J_{i+1}$. By contradiction:

$$
\Rightarrow \sum_{J_{h}^{-} \in h p\left(\pi_{j}, J_{i}^{-}\right)}^{L_{i+1}^{-}\left(\pi_{j}, t\right)} e_{j}^{-*}(t)>\sum_{J_{h} \in h p\left(\pi_{j}, J_{i}\right)} e_{j}^{*}(t)
$$

Since $\pi_{j}$ stay idle when higher priority jobs than $J_{i}^{-}$have finished their execution before their WCET, we obtain

$$
\sum_{J_{h}^{-} \in h p\left(\pi_{j}, J_{i}^{-}\right)} e_{j}^{-*}(t)=\sum_{J_{h} \in h p\left(\pi_{j}, J_{i}\right)} e_{j}^{*}(t)
$$

which is in contradiction with (2). The induction hypothesis follows.

LEMMA 2. Let $\tau$ denote any set of tasks that is schedulable by $\mathrm{r}-\mathrm{SP} \_\mathrm{wl}$. Let $\mathcal{J}$ denote a collection of jobs generated by $\tau$ The algorithm $\mathrm{r}-\mathrm{SP}$-wl meets all deadlines when scheduling any collection of jobs obtained from $\mathcal{J}$ by changing the parameters of one or more jobs by enlarging relative deadlines.

Proof. The scheduling decisions are made considering the laxity at time $t$ of the jobs. The deadline of the jobs is not used to compute this laxity then scheduling decision can not be changed if a deadline is enlarged.

LEMMA 3. Let $\tau$ denote any set of tasks that is schedulable by r-SP_wl. Let $\mathcal{J}$ denote a collection of jobs generated by $\tau$ The algorithm r-SP_wl meets all deadlines when scheduling any collection of jobs obtained from $\mathcal{J}$ by changing the parameters of one or more jobs by enlarging arrival times.

PROOF. The considered tasks are periodic then the arrival time of a job can not be increased.

Proposition 1. The static-priority restricted-migration scheduling algorithm $\mathrm{r}-\mathrm{SP}+\mathrm{wl}$ is sustainable with respect to WCET, relative deadlines and period.

Proof. The proof is made from Lemmas 1, 2 and 3.

\subsection{Sustainability of $r-S P_{-} w l$ tests}

In order to decide the schedulability of a set of tasks with $r$-SP_wl, we proposed a sufficient schedulability test based on the notion of $L O A D$. $L O A D$ at level $k$ represents the maximum processor demand of the jobs of priority greater than and equal to $k$ at any time $t$.

Theorem 1 (From [8]). Any set $\tau$ of periodic tasks satisfying:

$$
\forall k: 1 \leq k \leq n:\left[\operatorname{LOAD}(k) \leq \frac{1+(m-1) U_{\min }(k)}{1+2\left(D_{\max }(k) / D_{k}\right)}\right]
$$


is r-SP_wl schedulable upon identical multiprocessor platforms where $U_{\min }(k)$ is the minimum utilization and $D_{\max }(k)$ the maximum deadline of the tasks of priority from 1 to $k$.

The sufficient schedulability condition given by Theorem 1 is not sustainable. In order to decide schedulability of a set of tasks without sustainability problem, it is necessary to consider the schedule over the feasibility interval given by $\left[X_{1}, S_{n}+P\right]$ where $X_{n}$ is given by $X_{n}=S_{n}$ and $X_{i}=$ $O_{i}+\left\lfloor\frac{X_{i+1}-O_{i}}{T_{i}}\right\rfloor T_{i}$ and where $S_{n}$ is given by $S_{1}=O_{1}$ and $S_{i}=\max \left(O_{i}, O_{i}+\left\lceil\frac{S_{i-1}-O_{i}}{T_{i}}\right\rceil T_{i}\right) . \quad P$ denotes the hyperperiod of the set of tasks and $O_{i}$ the offset of the task $\tau_{i}$.

\section{CONCLUSION}

In the case of embedded real-time multiprocessor scheduling, the restricted-migration scheduling is an interesting approach. But it has been shown that it suffers from sustainability problems in the case of fixed-priority. In this paper, we review the notion of sustainability and we analyze a non-predictable algorithm in order to propose a sustainable alternative. We highlight the properties of lower-priorityagnostic test, processor-available assignment and work-conserving algorithm which are responsible of the non-predictability. We propose for our algorithm $r$-SP_wl the properties of lowerpriority-aware test, release-time assignment and idle algorithm. We prove that it does not suffer from scheduling anomaly with the model of tasks we consider.

In a previous work, we proposed a sufficient schedulability test for $r-S P_{-} w l$. Unfortunately, this test is not sustainable and we intend to develop a sustainable one in a future work.

\section{REFERENCES}

[1] B. Andersson, S. K. Baruah, and J. Jonsson. Static-priority scheduling on multiprocessors. In Proceedings of the 22nd IEEE Real-Time Systems Symposium (RTSS), pages 193-202, London, UK, December 2001. IEEE Computer Society.

[2] T. P. Baker. Comparison of empirical success rates of global vs. partitioned fixed-priority and EDF scheduling for hard real time. Technical Report TR-050601, Florida State University, Tallahassee, FL, USA, July 2005.

[3] T. P. Baker and S. K. Baruah. Sustainable multiprocessor scheduling of sporadic task systems. In Proceedings of the 21st Euromicro Conference on Real-time Systems (ECRTS), pages 141-150, Dublin, Irland, July 2009. IEEE Computer Society.

[4] S. K. Baruah and A. Burns. Sustainable scheduling analysis. In Proceedings of the 27th IEEE Real-Time Systems Symposium (RTSS), pages 159-168, Rio de Janeiro, Brazil, December 2006. IEEE Computer Society.

[5] S. K. Baruah and J. Carpenter. Multiprocessor fixed-priority scheduling with restricted interprocessor migrations. In Proceedings of the 15th Euromicro Conference on Real-time Systems (ECRTS), pages 195-202, Porto, Portugal, July 2003. IEEE Computer Society.

[6] A. Burns and S. K. Baruah. Sustainability in real-time scheduling. Journal of Computing Science and Engineering (JCSE), 2(1):74-97, March 2008.
[7] J. Carpenter, S. H. Funk, P. Holman, A. Srinivasan, J. H. Anderson, and S. K. Baruah. Handbook of Scheduling: Algorithms, Models, and Performance Analysis, chapter A Categorization of Real-Time Multiprocessor Scheduling Problems and Algorithms, pages 30-1 - 30-19. Chapman and Hall/CRC, Boca Raton, Florida, 2004.

[8] F. Fauberteau, S. Midonnet, and L. George. Laxity-based restricted-migration scheduling. In Proceedings of the 16th IEEE International Conference on Emerging Technologies and Factory Automation (ETFA), pages 1-8, Toulouse, France, September 2011. IEEE Computer Society.

[9] N. W. Fisher. The Multiprocessor Real-Time Scheduling of General Task Systems. PhD thesis, University of North Carolina at Chapel Hill, 2007.

[10] S. H. Funk. EDF Scheduling on Heterogeneous Multiprocessors. PhD thesis, University of North Carolina at Chapel Hill, 2004.

[11] L. George, P. Courbin, and Y. Sorel. Job vs. portioned partitioning for the earliest deadline first semi-partitioned scheduling. Journal of Systems Architecture, 57(5):518-535, May 2011.

[12] J. Goossens, S. H. Funk, and S. K. Baruah. Priority-driven scheduling of periodic task systems on multiprocessors. Real-Time Systems, 25(2-3):187-205, September 2003.

[13] R. Ha. Validating timing constraints in multiprocessor and distributed real-time systems. $\mathrm{PhD}$ thesis, University of Illinois, Dept. of Computer Science, Urbana-Champaign, IL, USA, 1995.

[14] R. Ha and J. W. S. Liu. Validating timing constraints in multiprocessor and distributed real-time systems. In Proceedings of the 14th International Conference on Distributed Computing Systems (ICDCS), pages 162-171, Pozman, Poland, June 1994. IEEE Computer Society.

[15] J. Y.-T. Leung and J. Whitehead. On the complexity of fixed-priority scheduling of periodic, real-time tasks. Performance Evaluation, 2(4):237-250, December 1982.

[16] C. L. Liu and J. W. Layland. Scheduling algorithms for multiprogramming in a hard-real-time environment. Journal of the ACM, 20(1):46-61, January 1973.

[17] J. M. López, M. García, J. L. Díaz, and D. F. García. Worst-case utilization bound for EDF scheduling on real-time multiprocessor systems. In Proceedings of the 12th Euromicro Conference on Real-time Systems (ECRTS), pages 25-33, Stockholm, Sweden, June 2000. IEEE Computer Society.

[18] A. K.-L. Mok. Fundamental Design Problems of Distributed Systems for Hard-Real-Time Environments. PhD thesis, Massachusetts Institute of Technology, Cambridge, MA, USA, May 1983.

[19] D.-I. Oh and T. P. Baker. Utilization bounds for $n$ -processor rate monotone scheduling with static processor assignment. Real-Time Systems, 15(2):183-192, September 1998. 